\title{
Flow-Cytometric Analysis of HNA-2 Expression and Phenotypes Among Thai Blood Donors
}

\author{
Oytip Nathalang, Ph.D. ${ }^{1}$, Kanokpol Siriphanthong, B.S. ${ }^{1}$, Sawang Petvises, Ph.D. ${ }^{2}$, and Nichapa Jeumjanya, M.S. ${ }^{3}$ \\ Graduate Program in Biomedical Sciences ${ }^{1}$ and Department of Medical Technology², Faculty of Allied Health Sciences, Thammasat University, Pathumtani, \\ Thailand; Blood Bank Section ${ }^{3}$, Thammasat University Hospital, Pathumtani, Thailand
}

Background: Antibodies specific to human neutrophil antigen (HNA), especially HNA-2, are implicated in various conditions, including neonatal alloimmune neutropenia, febrile non-hemolytic transfusion reactions, and transfusion-related acute lung injury. The distribution of the HNA-2 phenotype frequencies in the Thai population remains unknown. This study aimed to investigate HNA-2 phenotype frequencies in Thai blood donors and to compare the relationships of sex and age with HNA-2 expression.

Methods: EDTA blood samples were collected from 220 unrelated healthy Thai blood donors, including 150 males and 70 females, with ages ranging from 20 to 57 years. Polymorphonuclear cells were isolated and stained with monoclonal antibodies clone MEM166 and clone 2D1, which are specific to human CD177 (HNA-2) and CD45, respectively. HNA-2 expression according to sex and age was analyzed by flow cytometry.

Results: Among the 220 donors, HNA-2-positive and HNA-2-null-phenotype frequencies were 0.995 and 0.005 , respectively. Mean antigen expression was significantly higher in women $(71.01 \pm 15.46 \%)$ than in men $(64.59 \pm 18.85 \% ; P<0.05)$. No significant differences in HNA-2 expression were found between different age groups. HNA-2 phenotype frequencies were similar to those in Asian, African, American, and Brazilian populations, but were significantly different from those in eastern Japanese, Korean, and French populations $(P<0.001)$.

Conclusions: This is the first report of HNA-2 phenotype frequencies in a Thai population, and the data will be helpful in predicting the risk of HNA-2 alloimmunization and in recruiting granulocyte panel donors.

Key Words: HNA-2, Phenotype frequencies, Flow cytometry, Thais
Received: June 5, 2017

Revision received: August 1, 2017

Accepted: February 1, 2018

Corresponding author: Oytip Nathalang, Graduate Program, Faculty of Allied Health Sciences, Thammasat University, 99 Moo 18 Klongluang, Pathumtani 12120, Thailand

Tel: +66-29869213 Ext. 7231

Fax: $+66-25165379$

E-mail: oytipnt|@hotmail.com
() Korean Society for Laboratory Medicine This is an Open Access article distributed under the terms of the Creative Commons Attribution Non-Commercial License (http://creativecommons.org/licenses/by-nc/4.0) which permits unrestricted non-commercial use, distribution, and reproduction in any medium, provided the original work is properly cited.

\section{INTRODUCTION}

Antibodies specific to human neutrophil antigens (HNA), especially HNA-2 antibody, are implicated in alloimmune neonatal neutropenia (ANN), autoimmune neutropenia (AIN), transfusion-related acute lung injury (TRALI), drug-induced neutropenia, and graft failure after bone marrow transplantation [1, 2]. The HNA-2 antigen is located on the CD177 glycoprotein and is expressed specifically on neutrophils [1-3]. In HNA-2-positive individuals, two neutrophil subpopulations can be identified based on CD177 expression [4-6]. HNA-2-negative or HNA-2-null individuals do not express $\mathrm{CD} 177$ on neutrophils [7]. The frequency of HNA-2-positive individuals exceeds $90 \%$, while the HNA-2null phenotype varies between $0.0 \%$ and $2.0 \%$ among different populations. Interestingly, among eastern Japanese, Korean, and French populations, the frequency of HNA-2 null is $11.5 \%$ to $14.0 \%$ [8-10]. HNA-2 expression varies among individuals, ranging from $0 \%$ to $100 \%$, and is slightly higher in women than 
in men. HNA-2 expression decreases in elderly women, but not men [11, 12], and is reportedly increased in newborns, pregnant women, healthy people receiving granulocyte-colony-stimulating factor, and patients with severe bacterial infections and chronic myeloproliferative disorders [13-17].

Various techniques are used to detect HNA-2 antigen and antibodies. The combination of granulocyte agglutination test (GAT) and granulocyte immunofluorescence test (GIFT) is recommended for HNA antibody screening. Monoclonal antibodyspecific immobilization of granulocyte antigens (MAIGA) assay is used to confirm antibody specificities [2, 18]. However, the requirement for fresh granulocyte panels is a limitation of serological techniques. Regarding antigen detection, owing to the unavailability of specific antisera, various molecular techniques have been implemented to detect single nucleotide polymorphisms (SNPs) in HNA-1-, HNA-3-, HNA-4-, and HNA-5-encoding genes $[5,6]$. For the HNA-2 system, CD177 polymorphism is reportedly associated with different patterns of antigen expression; however, it remains inconclusive whether CD177 polymorphism determines HNA-2 deficiency [4, 7, 19-21]. Hence, molecular techniques are not able to distinguish HNA-2-positive and HNA-2-null individuals.

Flow cytometry is widely used for determining HNA-2 expression. This study aimed to investigate the HNA-2 phenotype frequencies among Thai blood donors by using a simple flow-cytometric analysis and to compare the relationships of sex and age with HNA-2 expression.

\section{METHODS}

\section{Study population}

This study involved 220 unrelated, healthy Thai blood donors who visited the blood bank of Thammasat University Hospital from November 2016 to April 2017, including 150 males and 70 females, with the median age of 38 years (range 20-57 years). Peripheral blood samples were collected in EDTA-anticoagulant tubes for HNA-2 expression analysis. Informed consent was obtained from each subject. This study was approved by the Committee on Human Rights Related to Research Involving Human Subjects, Thammasat University, Thailand (IRB No.132/2557).

\section{Antibodies}

Phycoerythrin (PE)-conjugated antibody specific for CD177 (clone MEM-166; BD Biosciences, Franklin Lakes, NJ, USA) and fluorescein isothiocyanate (FITC)-conjugated antibody specific for human CD45 (clone 2D1; BD Biosciences) were used for neutrophil staining. Isotype control antibodies including PEmouse IgG1, $\kappa$ (clone MOPC-21; BD Biosciences) and FITCmouse IgG1, $\kappa$ (clone MOPC-31C; BD Biosciences) were used to determine non-specific binding of antibodies. All antibodies were titrated before use to determine the concentration giving minimum saturation binding.

\section{Isolation of polymorphonuclear cells (PMNs)}

PMNs were isolated using Polymorphprep (AXIS-SHIELD; Alere Technologies AS, Oslo, Norway). Briefly, $3 \mathrm{~mL}$ of blood sample was overlaid on $3 \mathrm{~mL}$ of Polymorphprep in a 15-mL conical tube. Samples were centrifuged at $2,500 \mathrm{rpm}, 25^{\circ} \mathrm{C}$ for 35 minutes in a Universal 320/320R swing-out rotor centrifuge (Hettich Lab Technology, Tuttlingen, Germany). Following centrifugation, two layers, including a top layer of mononuclear cells and a lower layer of PMNs, separately appeared as white interfaces. The PMN layer was collected and the cells were washed twice with $2 \mathrm{~mL}$ of RPMI 1640 incomplete medium (Thermo Scientific, Waltham, MA, USA). To determine cell viability, the PMNs were stained with $0.4 \%$ trypan blue in phosphate-buffered saline $(\mathrm{pH}$ 7.2), and the number of viable cells was counted using a hemocytometer. At least $1 \times 10^{6}$ cells $/ \mathrm{mL}$ of PMNs was used to investigate HNA-2 expression.

\section{Antibody staining}

For antibody staining, $100 \mu \mathrm{L}$ of PMN suspension was incubated with $2.5 \mu \mathrm{L}$ of human CD177-PE and CD45-FITC antibodies on ice for 20 minutes. After incubation, the cells were washed twice with RPMI 1640 medium and centrifuged at 1,500 rpm, $4^{\circ} \mathrm{C}$ for 5 minutes. The supernatant was discarded, and the pellet was resuspended in $100 \mu \mathrm{L}$ RPMI 1640 medium for flow-cytometric analysis.

\section{Flow-cytometric analysis}

The stained neutrophils were analyzed for fluorescence intensity using a BD FACSVERSE flow cytometer (Becton Dickinson, Franklin Lakes, NJ, USA). Single-color controls of human CD177-PE and CD45-FITC antibodies were used to set compensation. In addition, isotype control antibodies were used to determine nonspecific binding of antibodies and to set gates. HNA-2 expression was analyzed using FlowJo software (FlowJo, Ashland, OR, USA). Variations in HNA-2 expression were observed by flow cytometry using the CD177 (MEM-166) antibody.

Total PMNs were initially defined by gating of CD45 and side scatter (SSC). Populations of CD45 high/SSC cells were further gated to determine the expression of CD177. According to pre- 
vious reports, an individual was regarded HNA-2-negative when less than $5 \%$ of neutrophils were reactive to the CD177 antibody $[12,20]$.

\section{Statistical analysis}

A two-tailed t-test was used to compare the expression of antigens among different sex and age groups. The subjects were grouped in four categories based on antigen expression, according to a previous report [20]: group 1, $\geq 60 \%$ (strong expression); group 2, 40-59.9\% (intermediate expression); group 3, 5-39.9\% (weak expression); and group $4,<5 \%$ (negative reaction). HNA-2 phenotype frequencies were compared with those previously reported for other populations by using a chi-square test [8-12, 22-25]. Statistical analyses were conducted using SPSS (Version 16.0, SPSS Inc., Chicago, IL, USA). A $P$ value $\leq 0.05$ was considered statistically significant.
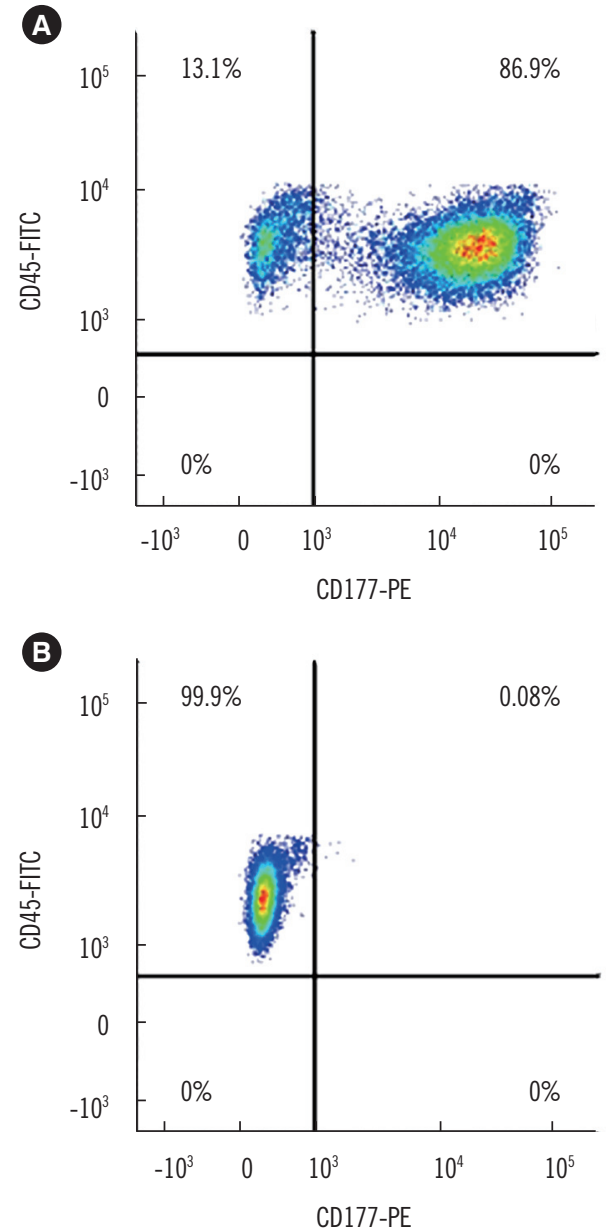

\section{RESULTS}

1. CD177 expression on neutrophils from healthy donors

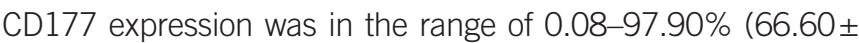
$18.10 \%$ ). Two populations of neutrophils in terms of CD177MEM-166 reactivity, with variation in HNA-2 expression, could be distinguished (Fig. 1). Among the 220 Thai blood donors, 219 were HNA-2 positive (99.55\%) and one male donor was HNA-2 negative (0.45\%).

\section{Effects of sex and age on HNA-2 expression}

Mean antigen expression was significantly higher in women $(71.01 \pm 15.46 \%)$ than in men $(64.59 \pm 18.85 \%)(t-t e s t=-2.669$; $95 \% \mathrm{Cl}:-11.17$ to $-1.70 ; \mathrm{P}<0.05)$. The distribution of HNA-2 expression showed no significant differences among age groups (Table 1).
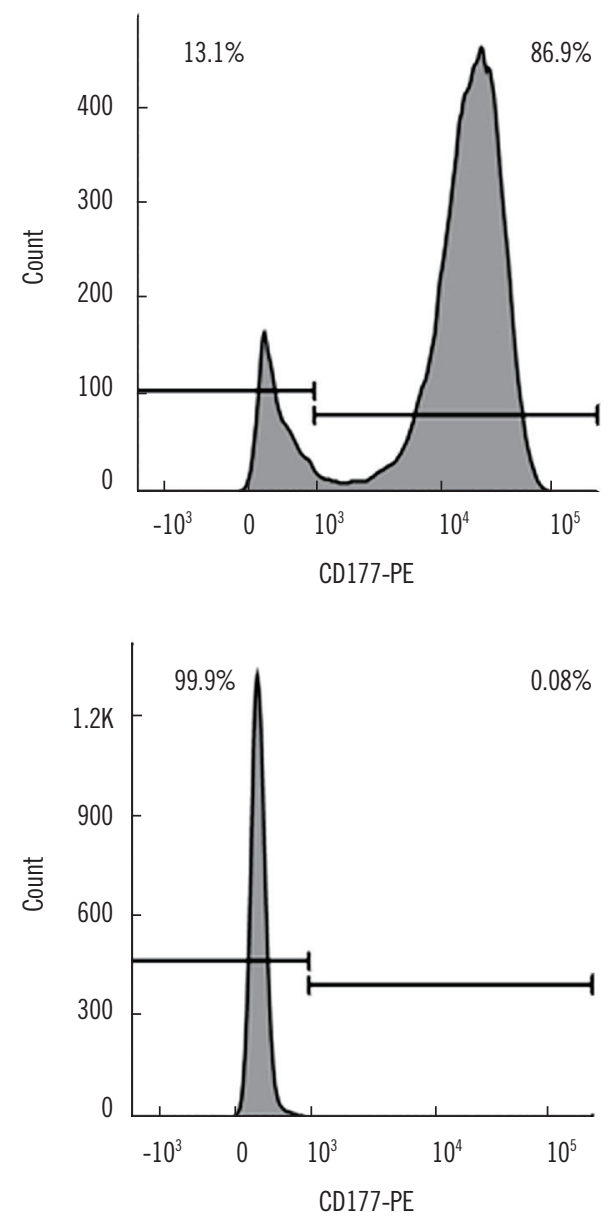

Fig. 1. Flow-cytometric analysis of the reactivity of CD45 and CD177 (MEM-166) with neutrophils. (A) HNA-2-positive individual showing 86.9\% HNA-2 expression; (B) HNA-2-negative individual showing 0.08\% HNA-2 expression. 
Table 1. Distribution of HNA-2 expression among healthy Thai blood donors stratified according to sex and age

\begin{tabular}{|c|c|c|c|c|c|c|}
\hline \multirow{2}{*}{ Sex } & \multirow{2}{*}{$\begin{array}{l}\text { Age } \\
\text { (year) }\end{array}$} & \multirow{2}{*}{$\mathrm{N}$} & \multicolumn{4}{|c|}{ HNA-2 expression, N (\%) } \\
\hline & & & $<5.0 \%$ & $5.0-39.9 \%$ & $40.0-59.9 \%$ & $\geq 60.0 \%$ \\
\hline \multirow[t]{4}{*}{ Female } & $17-35$ & 42 & $0(0.0)$ & 2 (2.9) & $9(12.9)$ & 31 (44.3) \\
\hline & $36-50$ & 24 & $0(0.0)$ & $1(1.4)$ & $5(7.1)$ & $18(25.7)$ \\
\hline & $\geq 51$ & 4 & $0(0.0)$ & $0(0.0)$ & $0(0.0)$ & $4(5.7)$ \\
\hline & Total & 70 & $0(0.0)$ & $3(4.3)$ & $14(20.0)$ & $53(75.7)$ \\
\hline \multirow[t]{4}{*}{ Male } & $17-35$ & 57 & $1(0.7)$ & $4(2.7)$ & $14(9.3)$ & $38(25.3)$ \\
\hline & $36-50$ & 67 & $0(0.0)$ & $7(4.7)$ & $18(12.0)$ & $42(28.0)$ \\
\hline & $\geq 51$ & 26 & $0(0.0)$ & $5(3.3)$ & $9(6.0)$ & $12(8.0)$ \\
\hline & Total & 150 & $1(0.7)$ & $16(10.7)$ & $41(27.3)$ & $92(61.3)$ \\
\hline
\end{tabular}

\section{Comparison of HNA-2 phenotype frequencies among populations}

HNA-2 phenotype frequencies in the Thai population were compared with those reported previously for Asian and other ethnic populations [8-12, 22-25] (Table 2). The HNA-2-positive phenotype had the highest incidence among populations, and its frequency in our study population was similar to that in Chinese, Taiwanese, Japanese, African, American, and Brazilian populations [11, 12, 22-25], but significantly higher than that in eastern Japanese, Korean, and French populations $(P<0.001)$ [8-10].

\section{DISCUSSION}

In this study, we developed a single-step method for HNA-2 phenotyping by flow cytometry using anti-human CD177 and CD45 monoclonal antibodies. Owing to the PMN preparation procedure used and CD45/SSC gating, monocyte contamination during neutrophil isolation was minimized. Additionally, two isotype control antibodies were used to determine non-specific antibody binding and to maximize the ability to discriminate between positive and negative neutrophil populations [26]. Hence, HNA-2positive and HNA-2-negative individuals could be identified according to the percentage of antigen expression ( $\geq 5 \%$ and $<5 \%$ ). Various CD177 antibodies have been used for HNA-2 phenotyping, including 1B5, TAG4, MEM-166, and 7D8 [11, 12, 27]. In this study using CD177 (MEM-166), we identified a single peak representing HNA-2-expressing neutrophils in flow-cytometric analysis. In contrast to previous reports, we identified no subpopulations with heterogeneous expression [11, 20, 27]. HNA-2 phenotyping using flow-cytrometric analysis has a few limitations in that the instrument is expensive and the blood samples need to be processed within 24 hours.
Table 2. HNA-2 phenotype frequencies among different populations

\begin{tabular}{|c|c|c|c|c|}
\hline \multirow{2}{*}{ Year } & \multirow{2}{*}{ Population } & \multirow{2}{*}{$\mathrm{N}$} & \multicolumn{2}{|c|}{ Phenotype frequency, $\mathrm{N}$ (\%) } \\
\hline & & & HNA-2-positive & HNA-2-negative \\
\hline 2017 [this study] & Thai & 220 & 219 (99.55) & $1(0.45)$ \\
\hline 1989 [8] & Eastern Japanese & 78 & $69(88.46)^{*}$ & $9(11.54)^{*}$ \\
\hline 1997 [9] & Korean & 105 & $90(85.71)^{*}$ & $15(14.29)^{*}$ \\
\hline 1990 [10] & French & 2,193 & $1,899(86.59)^{*}$ & $294(13.41)^{*}$ \\
\hline 2000 [11] & African & 54 & $53(98.15)$ & $1(1.85)$ \\
\hline 2000 [11] & American & 151 & $146(96.69)$ & $5(3.31)$ \\
\hline 2002 [12] & Western Japanese & 408 & 406 (99.51) & $2(0.49)$ \\
\hline 2011 [22] & Chinese & 83 & $83(100.00)$ & $0(0.00)$ \\
\hline 1994 [23] & Taiwanese & 128 & 127 (99.22) & $1(0.78)$ \\
\hline 2012 [24] & Japanese & 301 & 297 (98.67) & $4(1.33)$ \\
\hline 2009 [25] & Brazilian & 100 & $97(97.00)$ & $3(3.00)$ \\
\hline
\end{tabular}

*HNA-2-positive and -negative phenotype frequencies are different from those in Thai donors $(P<0.001)$.

Regarding the effects of sex and age on HNA-2 expression among the 220 Thai blood donors, HNA-2 expression was significantly higher in women than in men, in accordance with findings in Japanese and Brazilian populations [11, 12, 20]. These results revealed that estrogen levels might affect HNA-2 expression. In a Japanese population, HNA-2 expression reportedly decreased with age in women, while it remained constant in men [11]. In contrast, no association with age was found in both sexes in the current study, similar to previous findings in a Brazilian population [20]. The frequency of the HNA-2-positive phenotype resembled that in Asian, African, American, and Brazilian populations [11, 12, 22-25] and was significantly higher than that in eastern Japanese, Korean, and French populations [810]. This finding confirms that alloimmunization and clinical disorders, especially, ANN and TRALI, caused by antibodies against HNA-2 may be less probable than those caused by other HNA antibodies in Thais [28]. Further studies in unrelated healthy donors from different regions of Thailand are required to confirm this finding.

In conclusion, this is the first report of HNA-2 phenotype frequencies in a Thai population determined by flow-cytometric analysis. The data would be helpful in predicting the risk of HNA-2 alloimmunization and in recruiting granulocyte panel donors.

\section{Authors' Disclosures of Potential Conflicts of Interest}

No potential conflicts of interest relevant to this article were reported. 


\section{Acknowledgements}

This work was supported by grants of the National Research Council of Thailand and Thammasat University.

\section{REFERENCES}

1. Lalezari P, Murphy GB, Allen FH Jr. NB1, a new neutrophil-specific antigen involved in the pathogenesis of neonatal neutropenia. J Clin Invest 1971;50:1108-15.

2. Moritz E, Norcia AM, Cardone JD, Kuwano ST, Chiba AK, Yamamoto M, et al. Human neutrophil alloantigens systems. An Acad Bras Cienc 2009; 81:559-69.

3. Stroncek DF, Skubitz KM, McCullough JJ. Biochemical characterization of the neutrophil-specific antigen NB1. Blood 1990;75:744-55.

4. Wolff J, Brendel C, Fink L, Bohle RM, Kissel K, Bux J. Lack of NB1 GP (CD177/HNA-2a) gene transcription in NB1 GP- neutrophils from NB1 GP-expressing individuals and association of low expression with NB1 gene polymorphisms. Blood 2003;102:731-3.

5. Flesch BK. Human neutrophil antigens: a nomenclature update based on new alleles and new antigens. VOXS 2015;10(S1):243-9.

6. Muschter S, Berthold T, Greinacher A. Developments in the definition and clinical impact of human neutrophil antigens. Curr Opin Hematol 2011;18:452-60

7. Kissel K, Scheffler S, Kerowgan M, Bux J. Molecular basis of NB1 (HNA2a, CD177) deficiency. Blood 2002;99:4231-3.

8. Ohto $\mathrm{H}$ and Matsuo $\mathrm{Y}$. Neutrophil-specific antigens and gene frequencies in Japanese. Transfusion 1989;29:654.

9. Han KS and Um TH. Frequency of neutrophil-specific antigens among Koreans using the granulocyte indirect immunofluorescence test (GIFT). Immunohematology 1997;13:15-6.

10. Bierling P, Poulet E, Fromont P, Seror T, Bracq C, Duedari N. Neutrophil-specific antigen and gene frequencies in the French population. Transfusion 1990;30:848-9.

11. Matsuo K, Lin A, Procter JL, Clement L, Stroncek D. Variations in the expression of granulocyte antigen NB1. Transfusion 2000;40:654-62.

12. Taniguchi K, Kobayashi M, Harada H, Hiraoka A, Tanihiro M, Takata N, et al. Human neutrophil antigen-2a expression on neutrophils from healthy adults in western Japan. Transfusion 2002;42:651-7.

13. Wolff JC, Goehring K, Heckmann M, Bux J. Sex-dependent up regulation of CD 177-specific mRNA expression in cord blood due to different stimuli. Transfusion 2006;46:132-6.

14. Taniguchi K, Nagata H, Katsuki T, Nakashima C, Onodera R, Hiraoka A, et al. Significance of human neutrophil antigen-2a (NB1) expression and neutrophil number in pregnancy. Transfusion 2004;44:581-5.

15. Stroncek DF, Jaszcz W, Herr GP, Clay ME, McCullough J. Expression of neutrophil antigens after 10 days of granulocyte-colony-stimulating factor. Transfusion 1998;38:663-8.

16. Gohring K, Wolff J, Doppl W, Schmidt KL, Fenchel K, Pralle H, et al. Neutrophil CD177 (NB1 gp, HNA-2a) expression is increased in severe bacterial infections and polycythaemia vera. Br J Haematol 2004;126:252-4.

17. Passamonti F, Pietra D, Malabarba L, Rumi E, Della Porta MG, Malcovati $\mathrm{L}$, et al. Clinical significance of neutrophil CD177 mRNA expression in Ph-negative chronic myeloproliferative disorders. Br J Haematol 2004; 126:650-6.

18. Matsuhashi M, Tsuno NH, lino J, Nagura Y, Okazaki H, Santoso S. Advances in granulocyte test methodologies. ISBT Sci Ser 2014;9:246-52.

19. Li Y, Mair DC, Schuller RM, Li L, Wu J. Genetic mechanism of human neutrophil antigen 2 deficiency and expression variations. PLoS Genet 2015;11:e1005255

20. Moritz E, Chiba AK, Kimura EY, Albuquerque D, Guirão FP, Yamamoto M, et al. Molecular studies reveal that A134T, G156A and G1333A SNPs in the CD177 gene are associated with atypical expression of human neutrophil antigen-2. Vox Sang 2010;98:160-6.

21. Bayat B, Bein G, Sachs UJ. A sequence-specific polymerase chain reaction method for HNA-2 genotyping: homozygous c.843A>T mutation predicts the absence of CD177. Transfusion 2016;56:2127-32.

22. Xia W, Bayat B, Sachs U, Chen Y, Shao Y, Xu X, et al. The frequencies of human neutrophil alloantigens in the Chinese Han population of Guangzhou. Transfusion 2011;51:1271-7.

23. Lin M, Chen CC, Wang CL, Lee HL. Frequencies of neutrophil-specific antigens among Chinese in Taiwan. Vox Sang 1994;66:247.

24. Matsuhashi M, Tsuno NH, Kawabata M, Mishima Y, Okochi N, Santoso $\mathrm{S}$, et al. The frequencies of human neutrophil alloantigens among the Japanese population. Tissue Antigens 2012;80:336-40.

25. Norcia AM, Sugano EY, Chiba AK, Moritz E, Guirao FP, Yamamoto M, et al. Human neutrophil alloantigen-1a, $-1 \mathrm{~b},-2,-3 \mathrm{a}$ and $-4 \mathrm{a}$ frequencies in Brazilians. Tissue Antigens 2009;74:404-7.

26. Hulspas R, O'Gorman MR, Wood BL, Gratama JW, Sutherland DR. Considerations for the control of background fluorescence in clinical flow cytometry. Cytometry B Clin Cytom 2009;76:355-64.

27. Caruccio L, Walkovich K, Bettinotti M, Schuller R, Stroncek D. CD177 polymorphisms: correlation between high-frequency single nucleotide polymorphisms and neutrophil surface protein expression. Transfusion. 2004;44:77-82.

28. Changsri K, Tobunluepop P, Songthammawat D, Apornsuwan T, Kaset C, Nathalang $\mathrm{O}$. Human neutrophil alloantigen genotype frequencies in Thai blood donors. Blood Transfus 2014;12:s286-91. 